Research Article

\title{
Inactivation of SARS Coronavirus 2 and COVID-19 patient samples for contemporary immunology and metabolomics studies
}

Running title: Inactivation of COVID-19 samples

Devon J. Eddins ${ }^{a, b, c}$, Leda Bassit ${ }^{d, \dagger}$, Joshua D. Chandler ${ }^{b, e, \dagger}$, Natalie S. Haddad ${ }^{a, f}$, Katie L. Musall $^{d}$, Junkai Yang ${ }^{a}$, Astrid Kosters ${ }^{a}$, Brian S. Dobosh ${ }^{b, e}$, Mindy R. Hernández ${ }^{f}$, Richard P. Ramonell ${ }^{\mathrm{f}, \mathrm{g}}$, Rabindra M. Tirouvanziam ${ }^{\mathrm{b}, \mathrm{e}}$, F. Eun-Hyung Lee ${ }^{\mathrm{a}, \mathrm{c}, \mathrm{f}}$, Keivan Zandi ${ }^{\mathrm{d}}$, Raymond F. Schinazi ${ }^{d}$, Eliver E.B. Ghosn ${ }^{a, b, c^{*}}$

a Lowance Center for Human Immunology, Department of Medicine, Division of Immunology and Rheumatology, Emory University School of Medicine, Atlanta, GA 30322, USA

${ }^{b}$ Department of Pediatrics, Emory University School of Medicine, Atlanta, GA 30322, USA

${ }^{c}$ Emory Vaccine Center, Yerkes National Primate Research Center, Emory University School of Medicine, Atlanta, GA 30322, USA

'Center for AIDS Research, Laboratory of Biochemical Pharmacology, Department of Pediatrics, Emory University School of Medicine and Children's Healthcare of Atlanta, Atlanta, GA 30322, USA

e Center for CF and Airways Disease Research, Children's Healthcare of Atlanta and Department of Pediatrics, Emory University School of Medicine, Atlanta, GA, 30322, USA

${ }^{f}$ Department of Medicine, Division of Pulmonary, Allergy, Critical Care \& Sleep Medicine, Emory University School of Medicine, Atlanta, GA 30322, USA

g Current address: Department of Medicine, Division of Pulmonary, Allergy and Critical Care Medicine, University of Pittsburgh School of Medicine, Pittsburgh, PA 15213, USA

† These authors contributed equally: Leda Bassit and Joshua D. Chandler

\section{${ }^{*}$ Corresponding Author Contact Info}

Eliver E.B. Ghosn (ORCID: 0000-0001-7258-906X): Lowance Center for Human Immunology, Health Sciences Research Building, 1760 Haygood Dr. NE, E240, Atlanta, GA 30322, USA; tel: +1-404-712-3211; email: eliver.ghosn@emory.edu 


\section{Summary}

In late 2019, severe acute respiratory syndrome coronavirus 2 (SARS-CoV-2) emerged from Wuhan, China spurring the Coronavirus Disease-19 (COVID-19) pandemic that has resulted in over 219 million confirmed cases and nearly 4.6 million deaths worldwide. Intensive research efforts ensued to constrain SARS-CoV-2 and reduce COVID-19 disease burden. Due to the severity of this disease, the US Centers for Disease Control and Prevention (CDC) and World Health Organization (WHO) recommend that manipulation of active viral cultures of SARS-CoV2 and respiratory secretions from COVID-19 patients be performed in biosafety level 3 (BSL3) containment laboratories. Therefore, it is imperative to develop viral inactivation procedures that permit samples to be transferred and manipulated at lower containment levels (i.e., BSL2), and maintain the fidelity of downstream assays to expedite the development of medical countermeasures (MCMs). We demonstrate optimal conditions for complete viral inactivation following fixation of infected cells with paraformaldehyde solution or other commonly-used branded reagents for flow cytometry, UVC inactivation in sera and respiratory secretions for protein and antibody detection assays, heat inactivation following cDNA amplification of singlecell emulsions for droplet-based single-cell mRNA sequencing applications, and extraction with an organic solvent for metabolomic studies. Thus, we provide a suite of protocols for viral inactivation of SARS-CoV-2 and COVID-19 patient samples for downstream contemporary immunology assays that facilitate sample transfer to BSL2, providing a conceptual framework for rapid initiation of high-fidelity research as the COVID-19 pandemic continues.

56

\section{Keywords}

58 SARS-CoV-2, single-cell RNA-seq, flow cytometry, serology, metabolomics, 10X Genomics 59 


\section{Introduction}

At the end of 2019, a novel betacoronavirus, SARS-CoV-2 (1), emerged from Wuhan in the Hubei province of China causing viral pneumonia that progressed to severe or critical disease in $\sim 20 \%$ of infected patients, where in the most critical cases, patients would present with respiratory failure and require mechanical ventilator support in the intensive care unit (2-4). Since then, much research effort has been focused on better understanding pathogenesis and immunity to SARSCoV-2 (5). However, due to the severity of disease, work with infectious patient samples (primarily samples from the airways) and active viral cultures require biosafety level 3 (BSL3) containment facilities as with other closely related betacoronaviruses including SARS-CoV and Middle Eastern Respiratory Syndrome-Coronavirus (MERS-CoV) $(1,6,7)$. This can restrict research activity where containment facilities are not available. As the COVID-19 pandemic continues to surge with emergence of new variants (8), there is a continued need to conduct frontier research on COVID-19 immunology and pathogenesis to develop and refine medical counter measures (MCMs) to protect at risk populations and those disproportionately affected by COVID-19 disease (9-11). To facilitate this work, it is imperative to understand effective viral inactivation protocols that have minimal effects on assay readouts.

Though SARS-CoV-2 shares $~ 80 \%$ sequence homology with SARS-CoV (1), it is essential to evaluate efficacy of existing inactivation procedures on novel, independent viral strains. For example, MERS-CoV is also closely related to SARS-CoV but there are notable differences in the inactivation efficacy using gamma-irradiation between the viruses $(12,13)$. Indeed, there have been earlier reports of efficient viral propagation and inactivation procedures for SARS-CoV-2 using heat, fixatives/chemicals/surfactants (e.g., formaldehyde/Trizo ${ }^{\circledR} /$ Triton X-100), and UVC irradiation (14-19), which are comparable to SARS-CoV and MERS-CoV (12, 13, 20-22). However, though most of these studies highlight efficient and effective viral inactivation protocols (15-19), the effect of inactivation on the fidelity of downstream assay readouts and analysis remain largely unknown. Specifically, a detailed report on viral inactivation protocols and how they influence contemporary immunology assays is notably lacking. Contemporary immunological assays including ELISA/Luminex/Mesoscale assays for antibody/protein detection (9, 23-25), metabolomics $(26,27)$, high-dimensional (Hi-D) flow cytometry $(9,28-30)$, and multi-omics singlecell mRNA sequencing (scRNA-seq) $(9,31,32)$ are vital resources to develop and evaluate MCMs for the ongoing COVID-19 pandemic.

To address the need to successfully inactivate virus and permit transfer of material from BSL3 to a lower containment (i.e., BSL2) environment for high-fidelity downstream assays, we examined the efficiency of several viral inactivation methods for contemporary immunological assays including flow cytometry, serology/protein detection, scRNA-seq, and high-throughput metabolomic experiments using both culture-derived virus and infected respiratory samples from COVID-19 patients. Here, we report complete viral inactivation following fixation with $4 \%$ PFA or 1.6X BD FACS ${ }^{\text {TM }}$ Lysis Solution ( $2.5 \%$ formaldehyde and $~ 8.3 \%$ diethylene glycol) for 30 min at room temperature for flow cytometry, UVC inactivation at $\sim 4,000 \mu \mathrm{watts} / \mathrm{cm}^{2}$ for $30 \mathrm{~min}$ in sera and respiratory secretions for protein/antibody detection assays, heat inactivation following firstround cDNA amplification of single-cell emulsions for droplet-based scRNA-seq, and metabolite 
104

extraction for 30 min with 4 volumes of a 1:1 acetonitrile:methanol solution with $12.5 \mu \mathrm{M} / \mathrm{L}$ D5benzoylhippuric acid at $4{ }^{\circ} \mathrm{C}$ for metabolomic studies. These results will serve as conceptual framework and promote rapid initiation of cutting-edge immunology studies as the COVID-19 pandemic continues to evolve and for other risk group 3 agents that require higher containment.

\section{Methods}

\subsection{Ethics \& Biosafety statements}

COVID-19+ patients were recruited from the Intensive Care Units of Emory University, Emory St. Joseph's, Emory Decatur, and Emory Midtown Hospitals (severe) or the Emory Acute Respiratory Clinic (mild), and healthy adults were recruited from the Emory University Hospital. All studies were approved by the Emory Institutional Review Board (IRB) under protocol numbers IRB00058507, IRB00057983 and IRB00058271. Informed consent was obtained from the patients when they had decision making ability or from a legal authorized representative (LAR) if the patient was unable to provide consent. We collected both blood and non-induced sputum (healthy/mild) or endotracheal aspirate (ETA; severe). Study inclusion criteria included a confirmed COVID-19 diagnosis by PCR amplification of SARS-CoV-2 viral (v)RNA obtained from naso-/oro-pharyngeal swabs, age of 18 years or greater, and willingness and ability to provide informed consent. All work with infectious virus and respiratory samples from COVID-19 patients was conducted inside a biosafety cabinet within the Emory Health and Safety Office (EHSO)- and United States Department of Agriculture (USDA)-approved BSL3 containment facility in the Health Sciences Research Building at Emory University following protocols approved by the Institutional Biosafety Committee (IBC) and Biosafety Officer.

\subsection{Virus and cells}

African green monkey (Cercopithecus aethiops) kidney epithelial cells (Vero E6 cells; ATCC ${ }^{\circledR}$ CRL-1586 ${ }^{\mathrm{TM}}$ ) were maintained in complete (c)DMEM containing: 1X DMEM supplemented with $25 \mathrm{mM}$ HEPES, $2 \mathrm{mM}$ L-glutamine, $1 \mathrm{mM}$ sodium pyruvate, 1X non-essential amino acids (NEAA), $1 \mathrm{X}$ antibiotic/antimycotic solution (all from Corning) and 10\% heat-inactivated FBS (Gibco), unless indicated otherwise. Human lung adenocarcinoma epithelial cells (Calu-3 cells; ATCC ${ }^{\circledR}$ HTB$55^{\mathrm{TM}}$ ) were maintained in cMEM containing: $1 \mathrm{X}$ MEM (Corning) supplemented with $1 \mathrm{X}$ antibiotic/antimycotic solution and $10 \%$ heat-inactivated FBS unless indicated otherwise. Primary leukocytes from the airways of severe COVID-19 patients were collected bedside via endotracheal aspiration (ETA) and whole blood collected by standard venipuncture, then processed as previously described (9). SARS-CoV-2 USA-WA1/2020 (hereafter SCV2-WA1) was provided by BEI Resources (Manassas, VA, USA). Virus was propagated in Vero E6 cells as previously described $(16,33)$ and titer determined by $\operatorname{TCID}_{50}\left(\mathrm{TCID}_{50} / \mathrm{mL}\right)$ or plaque assays (PFU/mL). Low-passage (P1 or P2) virus stocks were used throughout this study.

\section{$2.3 \underline{\text { Infectivity assays }}$}

\subsubsection{Plaque Assays with Methylcellulose}

Vero E6 cells were seeded in 6-well plates (Falcon) with $5 \times 10^{5}$ cells/well in 5\% DMEM 24 h prior to infection and checked to verify $\geq 80 \%$ confluency. 10-fold dilutions of virus, respiratory secretions, and/or scRNA-seq emulsion in serum-free DMEM $(200 \mu \mathrm{L})$ were incubated on Vero 
E6 monolayers for $1 \mathrm{~h}$ absorption at $37^{\circ} \mathrm{C}$ with rocking at 15 min intervals. After absorption, cells were overlain with $2 \%$ methylcellulose (MilliporeSigma) in $2 \%$ DMEM for $72 \mathrm{~h}$ at $37^{\circ} \mathrm{C}$ in a $5 \%$ $\mathrm{CO}_{2}$, humidified incubator. $72 \mathrm{~h}$ post-infection (hpi), methylcellulose was carefully removed, and cells gently rinsed once with 1 X HBSS (Corning). Monolayers were fixed and plaques visualized with a solution of $0.4 \%$ crystal violet by weight in $80 \%$ methanol (MilliporeSigma) and $4 \%$ PFA (Electron Microscopy Sciences) for $20 \mathrm{~min}$ at room-temperature (RT).

\subsubsection{Plaque Assays with Agarose}

Vero E6 cells were seeded in 6 -well plates with $5 \times 10^{5}$ cells/well in $5 \%$ DMEM $24 \mathrm{~h}$ prior to infection and checked to verify $\geq 80 \%$ confluency. 10 -fold dilutions of virus, respiratory secretions, and/or scRNA-seq emulsion in serum-free DMEM (200 $\mu \mathrm{L})$ were incubated on Vero E6 monolayers for $1 \mathrm{~h}$ absorption at $37^{\circ} \mathrm{C}$ with rocking at $15 \mathrm{~min}$ intervals. After absorption, cells were overlain with $2 \mathrm{~mL} 0.5 \%$ immunodiffuse agarose (MP Biomedicals) in 1X DMEM supplemented with $5 \%$ FBS, $2 \mathrm{mM}$ L-glutamine, $1 \mathrm{mM}$ sodium pyruvate, 1X NEAA, 1X sodium bicarbonate, and $1 \mathrm{X}$ antibiotic/antimycotic solution. $72 \mathrm{hpi}$, a second $2 \mathrm{~mL}$ overlay of $0.5 \%$ immunodiffuse agarose in a 1 X HBSS solution with $0.026 \%$ neutral red (MilliporeSigma) was added for $\geq 3 \mathrm{~h}$ to visualize plaques.

\subsection{3 $\underline{T C I D}_{50}$ assays}

Vero E6 cells were seeded in 96-well plates with $2 \times 10^{4}$ cells/well in 5\% MEM $24 \mathrm{~h}$ prior to infection and checked to verify $\geq 80 \%$ confluency. 10 -fold dilutions of stock SCV2-WA1 virus in serum-free MEM $(100 \mu \mathrm{L})$ were incubated on Vero E6 monolayers in quadruplicates for $2 \mathrm{~h}$ absorption at $37^{\circ} \mathrm{C}$ without rocking. Following absorption, the inoculum was removed, and cells cultured in $2 \%$ MEM. Cells were assessed daily for cytopathic effect (CPE) compared to mockinfected negative controls by microscopy for $6 \mathrm{~d}$. Calculations for $50 \%$ tissue culture infectious dose (TCID 50 ) were performed using either the Spearman-Käber (34) or Reed and Muench (35) methods as previously described (36).

\subsubsection{Focus Reduction Neutralization Assays (FRNA)}

Vero E6 cells were seeded in 96-well plates with $2 \times 10^{4}$ cells/well in 5\% DMEM $24 \mathrm{~h}$ prior to infection and checked to verify $\geq 80 \%$ confluency. Dilutions of virus and/or virus treated with inactivation reagents in Opti-MEM ${ }^{\mathrm{TM}}(50 \mu \mathrm{L})$ were incubated on Vero E6 monolayers for $2 \mathrm{~h}$ absorption at $37^{\circ} \mathrm{C}$ without rocking. After absorption, cells were overlain with $2 \%$ methylcellulose in Opti-MEM ${ }^{\mathrm{TM}}$ (Gibco) supplemented with $2 \%$ FBS, $2.5 \mu \mathrm{g}$ amphotericin B (MilliporeSigma), and $20 \mu \mathrm{g} / \mathrm{mL}$ ciprofloxacin (MilliporeSigma) for $72 \mathrm{~h}$ at $37^{\circ} \mathrm{C}$ in a $5 \% \mathrm{CO}_{2}$, humidified incubator. 72 hpi, methylcellulose was carefully removed, and the cells fixed with 1:1 methanol/acetone mixture for 30 min at RT, then blocked with $200 \mu \mathrm{L} 5 \%$ milk in 1 X PBS for 20 min. Cells were incubated with an anti-SARS-CoV-2 spike RBD polyclonal antibody (Gentaur) at 1:3000 overnight at $37^{\circ} \mathrm{C}$. Cells were washed to remove excess antibody, then incubated with a secondary HRP-conjugated anti-human IgG for $1 \mathrm{~h}$ at $37^{\circ} \mathrm{C}$. Cells were washed to remove excess antibody and foci visualized using the TrueBlue ${ }^{\text {TM }}$ Peroxidase Substrate (SeraCare Life Sciences) incubated for $1 \mathrm{~h}$ at RT with rocking prior to imaging with an ELISpot reader for foci quantification. Data reported as focus forming units per $\mathrm{mL}(\mathrm{FFU} / \mathrm{mL})$. 


\subsection{Inactivation by fixative solutions}

Vero E6 cells were infected at a multiplicity of infection (MOI) of 0.01 and cultured in 6-well plates for $48 \mathrm{~h}$ with cMEM or cells from ETAs of COVID-19-infected patients were fixed with a freshly prepared $2 \%$ or $4 \%$ PFA solution (20\% stock diluted in 1X HBSS; Electron Microscopy Sciences) or a 1.6X BD FACS ${ }^{\mathrm{TM}}$ Lysis Solution (1:6 in sterile $\mathrm{dH}_{2} \mathrm{O}$; BD Biosciences) for the indicated time points at RT. Unfixed cells were incubated with $1 \mathrm{X}$ HBSS. Following fixation, cells from each condition were washed twice and resuspended in cMEM for an additional $48 \mathrm{~h}$ incubation at $37^{\circ} \mathrm{C}$ in a humidified, $5 \% \mathrm{CO}_{2}$ incubator. Culture supernatants were then collected and either plated immediately or frozen at $-80^{\circ} \mathrm{C}$ for analysis by plaque assay.

\subsection{Inactivation by ultraviolet $C$ (UVC) radiation} 50-1000 $\mu \mathrm{L}$ aliquots of SCV2-WA1 virus stock $\left(2.1 \times 10^{5} \mathrm{PFU} / \mathrm{mL}\right)$, respiratory supernatant (additional samples combined with our previously published data (9)), or patient sera were collected for UV inactivation. Samples were positioned 2-3 cm from the light source and exposed to $254 \mathrm{~nm}$ UVC light at maximum intensity $\left(\sim 4000 \mu \mathrm{watts} / \mathrm{cm}^{2}\right)$ for $30 \mathrm{~min}$ using a Spectrolinker ${ }^{\mathrm{TM}}$ $\mathrm{XL}-1000$ UV crosslinker (Spectronics Corporation) in either clear $2 \mathrm{~mL}$ microcentrifuge tubes (positioned on their side) or a 96-well plate (with the lid removed). Samples were either plated immediately or frozen at $-80^{\circ} \mathrm{C}$ for analysis by plaque assay.

\subsection{Inactivation for metabolomic assays}

To assess a nontoxic concentration of the metabolite extraction solvent ( $50 \%$ acetonitrile, $50 \%$ methanol, and $12.5 \mu \mathrm{M} / \mathrm{L}$ D5-benzoylhippuric acid) for subsequent FRNA, cytotoxicity tests were performed in Vero E6 cells via MTS assay using the CellTiter $96{ }^{\circledR}$ Non-Radioactive Cell Proliferation kit (Promega) as previously described (37). Uninfected Vero E6 cells were incubated with the extraction solvent, diluted in Opti-MEM ${ }^{\mathrm{TM}}$ at 1:10, 1:100, and 1:1000 in triplicate at $37^{\circ} \mathrm{C}$, $5 \% \mathrm{CO}_{2}$ for $72 \mathrm{~h}$ with 100,10 , and $1 \mu \mathrm{M}$ cycloheximide as positive control. After $72 \mathrm{~h}$, the MTS tetrazolium compound was added to the cells and incubated for an additional $2 \mathrm{~h}$. To determine the number of viable cells in each well, the absorbance was measured at $490 \mathrm{~nm}$ using a 96 -well plate reader (BioTek). Cytotoxicity was expressed as the dilution of the extraction solvent that inhibited cell proliferation by 50\% (IC50) and calculated using the Chou and Talalay method (38). SCV2-WA1 $\left(2.5 \times 10^{4} \mathrm{TCID}_{50} / \mathrm{mL}\right)$ was incubated with or without the extraction solvent (1:4) or Triton $\mathrm{X}-100$ for $30 \mathrm{~min}$ at $4^{\circ} \mathrm{C}$, then centrifuged at $20,000 \mathrm{xg}$ for $10 \mathrm{~min}$ at $4^{\circ} \mathrm{C}$. Supernatants were collected and diluted in Opti-MEM ${ }^{\mathrm{TM}}$ to final concentrations of 1:100 the extraction solvent or $1 \%$ Triton $\mathrm{X}-100$, and >100 FFU/mL SARS-CoV-2 per well for analysis by FRNA.

\subsection{Inactivation for scRNA-seq (10X Genomics)} encapsulated for scRNA-seq following the manufacturer's protocol "Chromium Next GEM Single Cell V(D)J Reagent Kits v1.1 User Guide with Feature Barcode technology for Cell Surface Protein" (document number CG000208; 10X Genomics) targeting 20,000 and 10,000 cells, respectively. An aliquot of the emulsion was collected following encapsulation for analysis by plaque assay. The remaining emulsion was processed for cDNA synthesis reaction following the manufacturer's protocol with reagent volumes adjusted to reflect the reduced reaction volume after taking aliquots for plaque assays. Polymerase chain reaction (PCR) amplification profile was 
$45 \mathrm{~min}$ at $53^{\circ} \mathrm{C}$ followed by $5 \mathrm{~min}$ at $85^{\circ} \mathrm{C}$. An additional aliquot of the cDNA suspension was collected following PCR reactions and either plated immediately or frozen at $-80^{\circ} \mathrm{C}$ for analysis by plaque assay. Plaque assays were also performed on the encapsulation emulsion alone (without including virus-infected cells) to evaluate reagent cytotoxicity on Vero E6 monolayers.

\subsection{Luminex proteomic serology assays}

Plasma from whole blood of COVID-19 patients was isolated via centrifugation at $400 \times \mathrm{g}$ for 10 $\min$ at $4^{\circ} \mathrm{C}$. To remove platelets, the isolated plasma was centrifuged at $4,000 \times \mathrm{g}$ for $10 \mathrm{~min}$ at $4^{\circ} \mathrm{C}$. Plasma samples were stored at $-80^{\circ} \mathrm{C}$ until analyzed. Luminex serology assays were performed as previously described (24). In brief, $\sim 50 \mu \mathrm{L}$ of coupled microsphere mix was added to each well of 96-well clear-bottom black polystyrene microplates (Greiner Bio-One) at a concentration of 1,000 microspheres per region per well. All wash steps and dilutions were performed with 1\% BSA in 1X PBS (hereafter assay buffer). Sera were assayed at 1:500 dilutions (in assay buffer) and surveyed for anti-SARS-CoV-2 N or RBD antibodies by $1 \mathrm{~h}$ incubation on a plate shaker at $800 \mathrm{rpm}$ in the dark. Following incubation, wells were washed five times with 100 $\mu \mathrm{L}$ of assay buffer using a BioTek 405 TS plate washer, then $3 \mu \mathrm{g} / \mathrm{mL}$ PE-conjugated goat antihuman $\lg \mathrm{A}$, IgG and/or IgM (Southern Biotech) was applied. After 30 min incubation, wells were washed three times in $100 \mu \mathrm{L}$ of assay buffer, then resuspended in $100 \mu \mathrm{L}$ of assay buffer for acquisition and analysis using a Luminex FLEXMAP 3D instrument and XPONENT 4.3 software (Luminex). Median fluorescent intensity (MFI) using combined or individual detection antibodies (i.e., anti-lgA, anti-lgG, or anti-lgM) was measured and the background value of assay buffer was subtracted from each serum sample result to obtain MFI minus background values (net MFI).

\subsection{Metabolomic assays}

Metabolites in human plasma were extracted from the National Institute of Standards and Technology (NIST) "Standard Reference Materials 1950" after mock or UVC treatment by addition of four volumes of $50 \%$ acetonitrile, $50 \%$ methanol, and $12.5 \mu \mathrm{M} / \mathrm{L}$ benzoyl-D5-hippuric acid (extraction solvent). Samples were vortexed for 10 seconds, incubated on ice for 30 min, then centrifuged at $20,000 \times \mathrm{g}$ for $10 \mathrm{~min}$ at $4^{\circ} \mathrm{C}$. The clear supernatant was aliquoted and injected $(2.5$ $\mu \mathrm{L}$ ) on a Vanquish Horizon liquid chromatograph coupled to $Q$ Exactive High Field (ThermoFisher). A $150 \mathrm{~mm} \times 2.1 \mathrm{~mm}$ ZIC-HILIC (MilliporeSigma) column and matching guard column were used to separate polar metabolites. Metabolites were ionized in both positive and negative mode and analyzed in full scan mode $(67-1000 \mathrm{~m} / \mathrm{z})$. Pooled quality control samples comprising equal proportions of every study sample were used to generate ddMS2 (Top20N) spectra of metabolites, evaluate assay reproducibility, and correct batch drift. Compound Discoverer 3.2 (ThermoFisher) was used to quantify peak areas and assign annotations based on a local library of reference standards or via matching metabolites to reference spectra in mzCloud (mzcloud.org). Data were imported to Prism 9 for graphing and statistical analyses by unpaired t-tests for untreated versus UVC-treated replicates, assuming individual variance, using the adaptive linear (two-step) step-up (Benjamini, Krieger, and Yekutieli) method (39) to control the false discovery rate (FDR), and a desired FDR (Q) of $10 \%$ for multiple comparisons.

\subsection{0 scRNA-seq data alignment, dimensionality reduction, and clustering}


The Cell Ranger Software (v.5.0.0; 10X Genomics) was used to perform cell barcode processing and single-cell 5' unique molecular identifier (UMI) counting. To detect SARS-CoV-2 reads, a customized reference genome was built by integrating human GRCh38 and SARS-CoV-2 genomes (severe acute respiratory syndrome coronavirus 2 isolate Wuhan-Hu-1, complete genome, GenBank MN908947.3). Splicing-aware aligner STAR (40) was used in FASTQ alignments. Cell barcodes were then determined based on the distribution of UMI counts automatically. The filtered gene-barcode matrices were first normalized using 'LogNormalize' method in Seurat v.3 (41) with default parameters. The top 2,000 variable genes were then identified using the 'vst' method by the 'FindVariableFeatures' function. Principal Component Analysis (PCA) was performed using the top 2,000 variable genes, then UMAPs generated using the top 30 principal components to visualize cells. Graph-based clustering was performed on the PCA-reduced data for clustering analysis with the resolution set to 0.8 to obtain the clusters. The total viral UMIs were the sum of the UMIs of the 12 SARS-CoV-2 genes (42).

\subsection{SARS-CoV-2 quantitative reverse transcription PCR (RT-qPCR)} Stock SCV2-WA1 virus and patient samples $(400 \mu \mathrm{L})$ were thoroughly mixed 1:1 with $2 \mathrm{X}$ DNA/RNA Shield ${ }^{\mathrm{TM}}$ and incubated at RT for $20 \mathrm{~min}$ for inactivation and vRNA was extracted using the Quick-RNA ${ }^{\mathrm{TM}}$ Viral Kit (Zymo Research) following the manufacturer's protocol. Complimentary (c)DNA was synthesized using the High-Capacity cDNA Reverse Transcription Kit (Applied Biosystems ${ }^{\mathrm{TM}}$ ) per manufacturer's instructions and diluted 1:5 in nuclease-free water, then $10 \mu \mathrm{L}$ of diluted cDNA was used with the NEB Luna Universal Probe qPCR Mastermix (New England BioLabs ${ }^{\circledR}$ ) following the manufacturer's protocol and RT-qPCR performed in 384-well plates using a QuantStudio ${ }^{\mathrm{TM}} 5$ Real-Time PCR System (Applied Biosystems ${ }^{\mathrm{TM}}$ ). Primer/probe pairs were: AGAAGATTGGTTAGATGATGATAGT (forward primer), TTCCATCTCTAATTGAGGTTGAACC (reverse primer), and /56-FAM/TCCTCACTGCCGTCTTGTTGACCA/3IABkFQ/ (probe), which were designed from sequences previously described (43) (Integrated DNA Technologies; IDT). To generate a standard curve and quantify SARS-CoV-2 genome copies, a gBlock with the sequence:

AATTAAGAACACGTCACCGCAAGAAGAAGATTGGTTAGATGATGATAGTCAACAAACTGTT GGTCAACAAGACGGCAGTGAGGACAATCAGACAACTACTATTCAAACAATTGTTGAGGTTC AACCTCAATTAGAGATGGAACTTACAGTTTCAGTGTTCAATTAA (IDT) was used as a standard.

To determine PFU equivalents (ePFU) from respiratory samples, vRNA was extracted from 10fold serial dilutions of stock SCV2-WA1 of a known titer for RT-qPCR to generate a standard curve from which number of genome copies per PFU could be extrapolated as previously described (44). Culture supernatants from mock- (1X HBSS) and SARS-CoV-2-infected Calu-3 ( $\mathrm{MOI} 0.04$ ) cells were utilized as additional controls.

\section{Results}

\subsection{Inactivation by fixation}

To evaluate the ability of commonly-used fixatives in flow cytometry (here, formaldehyde-based and PFA) to completely inactivate SARS-CoV-2-infected cells for transfer to lower containment 
settings, we performed a time-course of inactivation (Fig. 1A) using $2 \%$ and $4 \%$ PFA (diluted from a 20\% stock, see methods) along with 1.6X BD FACS ${ }^{\mathrm{TM}}$ Lysis Solution (10X stock diluted 1:6 in sterile $\mathrm{dH}_{2} \mathrm{O}$ ). Cells exposed to $2 \%$ and $4 \%$ PFA for $15 \mathrm{~min}$ at room temperature were still able to produce infectious virus when returned to culture for $48 \mathrm{~h}$ (Fig. 1B,C), with a decrease in viral titer $(\mathrm{PFU} / \mathrm{mL})$. This is in contrast to a previous report that indicated 10 min treatment with $4 \% \mathrm{PFA}$ is sufficient to inactivate virus (14). However, infectious virus was not detected by plaque assay following $15 \mathrm{~min}$ exposure to $1.6 \mathrm{X} \mathrm{BD} \mathrm{FACS}{ }^{\mathrm{TM}}$ lysis solution and at all subsequent time points (Fig. 1B,C). Cells treated with 4\% PFA for $30 \mathrm{~min}$ at RT were no longer infectious, however, 60 min was required to fully inactivate virus in cells treated with $2 \%$ PFA at RT. These data indicate that common fixation protocols (30 min fixation at RT) for commercially-available fixatives, specifically those with $\geq 4 \%$ PFA, are sufficient for inactivating SARS-CoV-2 infected cells.

\subsection{UVC inactivation}

\subsubsection{Inactivation of respiratory secretions and viral stocks}

Though complete inactivation of SARS-CoV viral stocks can be achieved with $<15$ min exposure to UVC-irradiation (12), a follow-up report for SARS-CoV inactivation in non-cellular blood products in PBS solutions recommended 40 min exposure to inactivate virus (20). Therefore, we selected 30 min exposure to UVC-irradiation at maximum intensity $\left(\sim 4000 \mu \mathrm{watt} / \mathrm{cm}^{2}\right)$ for both culture-derived SARS-CoV-2 viral stocks and respiratory secretions from COVID-19 patients. First, we determined viral load in the respiratory secretions by extrapolating ePFU/mL from RTqPCR results of respiratory secretions (see methods) (44). Using a standard curve generated from virus stock of a known titer (Fig. 2A), we determined 1 PFU to be equivalent to 73 SARSCoV-2 genome copies in RT-qPCR data. This allowed for more accurate viral detection and quantification (Fig. 2B) since endotracheal aspirate (ETA) and bronchoalveolar lavage fluid (BALF) samples are standard modalities used to diagnose ventilator-associated pneumonia (45, 46) and can be inundated with pulmonary microbes that grow in cultures, confounding traditional plaque assays (see Fig. S1). As expected from our previous study (9), we found that viral load varied across patient groups where those with severe disease had lower (or absent) viral load at time of sampling (samples were combined with our previously data (9); Fig. 2B). Following 30 min exposure to UVC-irradiation, both virus stock $\left(2.1 \times 10^{5} \mathrm{PFU} / \mathrm{mL}\right)$, and respiratory secretions were not detected by plaque assay (Fig. $3 \mathrm{C}$ ), indicating complete viral inactivation. Additionally, we demonstrate that UVC-inactivation abolishes microbial growth in plaque assays that hampered accurate viral load detection in ETA samples (Fig. S1), which is consistent with established efficacy of UVC-inactivation for a diverse range of pathogenic microbes $(47,48)$.

\subsubsection{Effects on antibody measurements}

To determine the effect of 30 min exposure to UVC-irradiation on protein/antibody detection, we compared untreated and UVC-treated plasma samples from both mild and severe COVID-19 patients (Fig. 2D). We did not observe any significant differences in the levels of anti-SARS-CoV2 antibodies detected by Luminex proteomic assays. Collectively, these data indicate that $30 \mathrm{~min}$ exposure to UVC-irradiation at maximum intensity $\left(\sim 4000 \mu \mathrm{watt} / \mathrm{cm}^{2}\right)$ is both effective on inactivating high titer stock SARS-CoV-2 and respiratory secretions from COVID-19 patients, with minimal effect on downstream protein/antibody assays. 


\subsection{Inactivation for metabolomics}

\subsubsection{UVC treatment}

To determine optimal inactivation procedure for metabolomics, we first evaluated the effects of UVC-inactivation described above on Standard Reference Material 1950 of metabolites in human plasma (49). UVC-inactivation significantly altered the metabolic profile of samples (Fig. 3A). We show that the differentially-expressed metabolites between untreated and UVC-treated plasma samples are redox active metabolites (Fig. 3B), suggesting that reactive oxygen species (ROS) known to be produced during UVC irradiation (50) lead to sample oxidation during this procedure - similar to ROS oxidation in vivo (51-53). Therefore, UVC-inactivation is not suitable for metabolomic studies, especially if interrogating redox active metabolites.

\subsubsection{Treatment with organic solvents}

We then wanted to test if the standard metabolomic sample extraction procedure with organic solvents (see methods), could successfully inactivate SARS-CoV-2-infected non-cellular products (such as respiratory secretions or plasma) as an alternative to UVC-inactivation. Specifically, we used a 1:1 mixture of acetonitrile and methanol including a deuterated internal standard, administered at 4 volumes relative to starting sample. We performed FRNAs with stock SCV2WA1 (untreated), and virus treated with either the extraction solvent or Triton X-100, which has been shown to inactivate SARS-CoV-2 $(17,18)$. Incubation of SCV2-WA1 virus stock 1:4 with the extraction solvent was sufficient to fully inactivate virus along with Triton X-100 (Fig. 3C). Cells incubated with the extraction solvent remained viable (Fig. $3 \mathrm{C}$ ), confirming virus inactivation independent of Vero E6 cytotoxicity, which was observed for other chemical reagents/surfactant such as Triton X-100 (Fig. 3C). Therefore, these results demonstrate that the standard metabolomic assay sample processing procedure with 4 volumes of our extraction solvent is sufficient to inactivate SARS-CoV-2-infected non-cellular samples while retaining sample integrity as compared to UVC-inactivation.

\subsection{Inactivation for scRNA-seq}

To better understand efficacy of SARS-CoV-2 inactivation in droplet-based scRNA-seq pipelines-specifically the 10X Genomics platform - we evaluated viral inactivation at the two early steps in the manufacturer's instructions. First, we evaluated the reagent cytotoxicity of the 10X Genomics encapsulation emulsion on Vero E6 cells by performing plaque assays with the emulsion free of encapsulated cells. We demonstrate that the reagents in the emulsion (many of which are proprietary) are not inherently cytotoxic to Vero E6 monolayers, allowing us to evaluate viral inactivation by standard plaque assay (Fig.4a).

We next evaluated the efficacy of viral inactivation following single-cell encapsulation (which contains a proprietary lysis solution) of SCV2-WA1-infected (MOI 0.04) Vero E6 or Calu-3 cells. We show that the standard single-cell encapsulation step alone is not sufficient to fully inactivate SARS-CoV-2, which could be detected in subsequent plaque assays (Fig. 4A,B). Therefore, we tested the efficacy of the first round cDNA synthesis reaction, which includes exposure to temperatures $\geq 53^{\circ} \mathrm{C}$, in viral inactivation. We show that after the PCR reaction $\left(45 \mathrm{~min}\right.$ at $53^{\circ} \mathrm{C}$ followed by $5 \mathrm{~min}$ at $85^{\circ} \mathrm{C}$ ), infectious SARS-CoV-2 was not detectable by plaque assay (Fig. 
4A,B). Taken together, our data demonstrate that scRNA-seq emulsions of SARS-CoV-2-infected cells are fully inactivated only after heat inactivation following the cDNA synthesis reaction using the conditions described in the manufacturer's protocol $\left(45 \mathrm{~min}\right.$ at $53^{\circ} \mathrm{C}$ followed by $5 \mathrm{~min}$ at $85^{\circ} \mathrm{C}$ ), which can then be transferred to lower containment for library preparation and sequencing. Indeed, after sequencing, we find $>2.3$ million viral transcripts (or unique molecular identifiers; UMI) in 8,000 SCV2-WA1-infected Calu-3 cells (Fig. 4C).

\section{Discussion}

To date, there have been multiple studies to evaluate efficacy of viral inactivation procedures using heat, chemicals, and UVC irradiation on SARS-CoV-2-infected samples (14-18). Many of these studies have evaluated traditional procedures established for SARS-CoV and MERS-CoV and were found to have comparable efficacies $(12,13,20,21)$. Here, we add to these studies by evaluating inactivation procedures performed under optimized conditions that allow for downstream processing/analysis for contemporary immunology assays with limited effects on assay readouts. We caution that all procedures are performed under the specified conditions and those that differ from what have been described here should be evaluated on viral stocks and patient samples before transferring to lower biosafety containment.

Since its induction in the 1960s, flow cytometry has been the preeminent technology for singlecell analysis (54) particularly for investigating the heterogeneity of the immune system in health and disease $(55,56)$. Indeed, Hi-D flow cytometry has been a pivotal tool in dissecting the complex immunophenotypes of leukocytes in COVID-19 $(9,29,30,57,58)$. We evaluated the ability of commercially-available fixatives commonly used in flow cytometry (formaldehyde-based) to fully inactivate SARS-CoV-2-infected cells to facilitate transfer of cells from BSL3 to BSL2 for data acquisition (9). Here, we show that treatment with 4\% PFA or 1.6X BD FACS ${ }^{\text {TM }}$ Lysis solution for 30 min at RT was sufficient to completely inactivate SARS-CoV-2-infected cells, even at viral titers higher than in cells from infected patients. Therefore, most common fixation protocols and reagents (e.g., BD Cytofix/Cytoperm ${ }^{\mathrm{TM}}$, BioLegend ${ }^{\circledR}$ Fixation Buffer, etc.) that contain $\geq 4 \%$ PFA are suitable for preparing fluorescently-stained, SARS-CoV-2-infected cells for transfer out of BSL3 containment after 30 min exposure. Conversely, lower concentrations of PFA (i.e., 2\%) required longer exposure time (at least $60 \mathrm{~min}$ at $\mathrm{RT}$ ) to fully inactivate samples.

Similarly, we show that UVC irradiation $\left(\sim 4000 \mu \mathrm{watts} / \mathrm{cm}^{2}\right)$ for $30 \mathrm{~min}$ is sufficient to fully inactivate high titer SCV2-WA1 viral stocks and respiratory supernatants from patients with minimal effects on protein/antibody detection (59), which will promote further studies on secretions from ETA and/or BALF to better understand local versus systemic responses $(9,25)$. It is important to note that a previous study on UVC-inactivation of SARS-CoV found BSA to protect virus from UVC-inactivation even after 60 min exposure (20). Therefore, we avoided BSA in solutions used in respiratory sample preparation (9), and plasma samples were inactivated prior to dilution in the Luminex proteomic assay buffer (1\% BSA in 1X PBS, see methods). significantly altered metabolomic profiles is human plasma samples. Specifically, we show that 
redox active metabolites such as methionine and urate are oxidized following UVC inactivation, significantly increasing signals for methionine sulfoxide and allantoin, respectively $(51,52)$. Similarly, bilirubin, which is oxidized to biliverdin, is significantly decreased with UVC treatment (53). Therefore, UVC-inactivation of clinical samples could lead to misleading biological interpretations, artificially skewing sample metabolites to a more oxidized profile (51-53). However, high concentration methanol $(\geq 80 \%)(60,61)$ and methanol/acetone mixtures $(13,22)$ have previously been shown to successfully inactivate many viral infected samples including SARS-CoV-2 (17-19). The extraction solvent we used is similar to that used in many metabolomic sample preparation techniques, and was sufficient to inactivate virus while maintaining data integrity.

Systems immunology approaches, including multi-omic scRNA-seq, have greatly advanced our understanding of COVID-19 immunity and pathogenesis $(9,31,32)$. However, a detailed report on inactivation efficacy of scRNA-seq pipelines is notably lacking. We demonstrate that in the standard 10X Genomics pipeline, encapsulation alone was insufficient to fully inactivate virus. According to the manufacturer's guidelines, a cDNA synthesis reaction is the next immediate step after encapsulation (see methods). Though many studies have evaluated the efficacy of heat inactivation for SARS-CoV-2 and demonstrated $45 \mathrm{~min}$ at $56^{\circ} \mathrm{C}$ and $5 \mathrm{~min}$ at $100^{\circ} \mathrm{C}$ are sufficient to fully inactivate virus (14-19), none have tested the specific conditions for the cDNA synthesis reaction ( $45 \mathrm{~min}$ at $53^{\circ} \mathrm{C}$ followed by $5 \mathrm{~min}$ at $85^{\circ} \mathrm{C}$ ). Here, we expand on the previous studies by demonstrating that the cDNA synthesis reaction in the standard 10X Genomics pipeline successfully inactivates SARS-CoV-2 and allows for transfer to lower containment for subsequent processing and library generation procedures.

Thus, we report optimized methods of viral inactivation that have minimal, if any, adverse impact on immunological studies of infected culture-derived and patient samples, permitting safe transfer to lower containment laboratories (BSL2) for final processing and data acquisition. Taken together, this suite of inactivation procedures can serve as guidelines for rapid initiation of research as the COVID-19 pandemic continues. 


\section{Acknowledgements}

This work was supported by funds from NIH/NIAID R01Al123126 (EEBG) and R01Al12312605S1 (EEBG), NIH T32-HL116271-07 (RPR), the Program for Breakthrough Biomedical Research, and Lowance Center for Human Immunology (EEBG), the NSF EAGER award 2032273 (RT, RFS, KZ), and the Woodruff Health Science Center COVID-19 CURE award (RT, RFS, KZ). DJE was supported, in part, by the Laney Graduate School Fellowship (Emory) and JDC was supported, in part, by CF@LANTA, a component of Emory University and Children's Healthcare of Atlanta. The funders had no role in study design, data collection and analysis, decision to publish, or preparation of the manuscript. We thank Fred Souret (10X Genomics) for providing additional reaction kits to test viral inactivation in scRNA-seq assays, Doan Nguyen for technical direction with the serology experiments, and the Emory Pediatric/Winship Flow Cytometry Core (access supported in part by Children's Healthcare of Atlanta) for their support with flow cytometry experiments. We thank Rafi Ahmed (Emory) and Jacob Kohlmeier (Emory) for kindly providing the Vero E6 and Calu-3 cell lines, respectively. We thank Ann Chahroudi, Nils Schoof, Kira Moresco, and Stacy Heilman of the Department of Pediatrics (Emory), along with the Emory Biosafety Officers Kalpana Rengarajan and Esmeralda Meyer and the Institutional Biosafety Committee (IBC) for their assistance with the BSL3 facility and protocol review/approval.

\section{Declaration of interest}

FEL is the founder of MicroB-plex, Inc., serves on the SAB of Be Bio Pharma, receives grants from BMGF and Genentech, and receives royalties from BLI, inc. All other authors have no 510 competing interest to declare. 


\section{Author contributions}

Devon J. Eddins: Conceptualization, Data curation, Formal analysis, Investigation, Methodology,

514 Validation, Visualization, Writing - original draft, Revising \& editing - final draft. Leda Bassit:

515 Investigation, Methodology, Data curation, Formal Analysis, Visualization. Joshua D. Chandler:

516 Investigation, Methodology, Data curation, Formal Analysis, Visualization. Natalie S. Haddad

517 Investigation, Data curation, Formal Analysis. Katie L. Musall: Investigation. Junkai Yang:

518 Formal Analysis, Visualization. Astrid Kosters: Investigation. Brian S. Dobosh: Investigation,

519 Formal Analysis. Mindy R. Hernández: Resources. Richard P. Ramonell: Resources. Rabindra

520 M. Tirouvanziam: Supervision, Resources, Data curation. F. Eun-Hyung Lee: Supervision, 521 Resources, Data curation. Keivan Zandi: Resources, Investigation, Methodology, Data curation, 522 Formal analysis, Supervision. Raymond F. Schinazi: Supervision, Resources, Data curation.

523 Eliver E.B. Ghosn: Conceptualization, Data curation, Formal analysis, Funding acquisition, 524 Methodology, Project administration, Resources, Supervision, Visualization, Writing - original 525 draft; Revising \& editing - final draft. All authors discussed the results and read and approved the 526 final manuscript. 


\section{Literature Cited}

1. Gorbalenya AE, Baker SC, Baric RS, de Groot RJ, Drosten C, Gulyaeva AA, et al. The species Severe acute respiratory syndrome-related coronavirus: classifying 2019-nCoV and naming it SARS-CoV-2. Nat Microbiol. 2020 Apr;5(4):536-44.

2. Huang C, Wang Y, Li X, Ren L, Zhao J, Hu Y, et al. Clinical features of patients infected with 2019 novel coronavirus in Wuhan, China. Lancet. 2020 Feb 15;395(10223):497-506.

3. Merad M, Martin JC. Pathological inflammation in patients with COVID-19: a key role for monocytes and macrophages. Nat Rev Immunol. 2020 Jun;20(6):355-62.

4. Wu C, Chen X, Cai Y, Xia J, Zhou X, Xu S, et al. Risk Factors Associated With Acute Respiratory Distress Syndrome and Death in Patients With Coronavirus Disease 2019 Pneumonia in Wuhan, China. JAMA Intern Med. 2020 Jul 1;180(7):934-43.

5. Carvalho T, Krammer F, Iwasaki A. The first 12 months of COVID-19: a timeline of immunological insights. Nat Rev Immunol. 2021 Apr;21(4):245-56.

6. de Wit E, van Doremalen N, Falzarano D, Munster VJ. SARS and MERS: recent insights into emerging coronaviruses. Nat Rev Microbiol. 2016 Aug;14(8):523-34.

7. Graham RL, Donaldson EF, Baric RS. A decade after SARS: strategies for controlling emerging coronaviruses. Nat Rev Microbiol. 2013 Dec;11(12):836-48.

8. Harvey WT, Carabelli AM, Jackson B, Gupta RK, Thomson EC, Harrison EM, et al. SARS-CoV-2 variants, spike mutations and immune escape. Nat Rev Microbiol. 2021 Jul;19(7):409-24.

9. Eddins DJ, Yang J, Kosters A, Giacalone V, Pechuan X, Chandler JD, et al. Pathogenic neutrophilia drives acute respiratory distress syndrome in severe COVID-19 patients. bioRxiv. 2021.

10. Romano SD, Blackstock AJ, Taylor EV, El Burai Felix S, Adjei S, Singleton CM, et al. Trends in Racial and Ethnic Disparities in COVID-19 Hospitalizations, by Region - United States, March-December 2020. MMWR Morb Mortal Wkly Rep. 2021 Apr 16;70(15):560-5. 11. Van Dyke ME, Mendoza MCB, Li W, Parker EM, Belay B, Davis EM, et al. Racial and Ethnic Disparities in COVID-19 Incidence by Age, Sex, and Period Among Persons Aged <25 Years - 16 U.S. Jurisdictions, January 1-December 31, 2020. MMWR Morb Mortal Wkly Rep. 2021 Mar 19;70(11):382-8.

12. Darnell ME, Subbarao K, Feinstone SM, Taylor DR. Inactivation of the coronavirus that induces severe acute respiratory syndrome, SARS-CoV. J Virol Methods. 2004 Oct;121(1):8591.

13. Kumar M, Mazur S, Ork BL, Postnikova E, Hensley LE, Jahrling PB, et al. Inactivation and safety testing of Middle East Respiratory Syndrome Coronavirus. J Virol Methods. 2015 Oct;223:13-8.

14. Case JB, Bailey AL, Kim AS, Chen RE, Diamond MS. Growth, detection, quantification, and inactivation of SARS-CoV-2. Virology. 2020 Sep;548:39-48.

15. Inagaki $H$, Saito A, Sugiyama $H$, Okabayashi T, Fujimoto S. Rapid inactivation of SARSCoV-2 with deep-UV LED irradiation. Emerging Microbes \& Infections. 2020;9(1):1744-7.

16. Jureka AS, Silvas JA, Basler CF. Propagation, Inactivation, and Safety Testing of SARSCoV-2. Viruses. 2020 Jun 6;12(6).

17. Patterson El, Prince T, Anderson ER, Casas-Sanchez A, Smith SL, Cansado-Utrilla C, et al. Methods of Inactivation of SARS-CoV-2 for Downstream Biological Assays. J Infect Dis. 2020 Oct $1 ; 222(9): 1462-7$.

18. Welch SR, Davies KA, Buczkowski H, Hettiarachchi N, Green N, Arnold U, et al. Analysis of inactivation of SARS-CoV-2 by specimen transport media, nucleic acid extraction reagents, detergents, and fixatives. Journal of clinical microbiology. 2020;58(11). 
19. Widera M, Westhaus S, Rabenau HF, Hoehl S, Bojkova D, Cinatl J, Jr., et al. Evaluation of stability and inactivation methods of SARS-CoV-2 in context of laboratory settings. Med Microbiol Immunol. 2021 Aug;210(4):235-44. 20. Darnell ME, Taylor DR. Evaluation of inactivation methods for severe acute respiratory syndrome coronavirus in noncellular blood products. Transfusion. 2006 Oct;46(10):1770-7. 21. Rabenau HF, Cinatl J, Morgenstern B, Bauer G, Preiser W, Doerr HW. Stability and inactivation of SARS coronavirus. Med Microbiol Immunol. 2005 Jan;194(1-2):1-6.

22. Kariwa H, Fujii N, Takashima I. Inactivation of SARS coronavirus by means of povidoneiodine, physical conditions and chemical reagents. Dermatology. 2006;212 Suppl 1(Suppl

588 23. Clemente A, Alba-Patiño A, Santopolo G, Rojo-Molinero E, Oliver A, Borges M, et al. Immunodetection of Lung IgG and IgM Antibodies against SARS-CoV-2 via Enzymatic Liquefaction of Respiratory Samples from COVID-19 Patients. Anal Chem. 2021 Mar 30;93(12):5259-66.

24. Haddad NS, Nguyen DC, Kuruvilla ME, Morrison-Porter A, Anam F, Cashman KS, et al. One-Stop Serum Assay Identifies COVID-19 Disease Severity and Vaccination Responses. ImmunoHorizons. 2021;5(5):322-35. 25. Sterlin D, Mathian A, Miyara M, Mohr A, Anna F, Claër L, et al. IgA dominates the early neutralizing antibody response to SARS-CoV-2. Sci Transl Med. 2021 Jan 20;13(577).

26. Bernatchez JA, McCall LI. Insights gained into respiratory infection pathogenesis using lung tissue metabolomics. PLoS Pathog. 2020 Jul;16(7):e1008662.

27. Olszewski KL, Morrisey JM, Wilinski D, Burns JM, Vaidya AB, Rabinowitz JD, et al. Host-parasite interactions revealed by Plasmodium falciparum metabolomics. Cell Host Microbe. 2009 Feb 19;5(2):191-9.

28. Brummelman J, Haftmann C, Núñez NG, Alvisi G, Mazza EMC, Becher B, et al. Development, application and computational analysis of high-dimensional fluorescent antibody panels for single-cell flow cytometry. Nat Protoc. 2019 Jul;14(7):1946-69.

29. Lourda M, Dzidic M, Hertwig L, Bergsten H, Palma Medina LM, Sinha I, et al. Highdimensional profiling reveals phenotypic heterogeneity and disease-specific alterations of granulocytes in COVID-19. Proc Natl Acad Sci U S A. 2021 Oct 5;118(40).

30. Mathew D, Giles JR, Baxter AE, Oldridge DA, Greenplate AR, Wu JE, et al. Deep immune profiling of COVID-19 patients reveals distinct immunotypes with therapeutic implications. Science. 2020 Sep 4;369(6508).

31. Stephenson E, Reynolds G, Botting RA, Calero-Nieto FJ, Morgan MD, Tuong ZK, et al. Single-cell multi-omics analysis of the immune response in COVID-19. Nat Med. 2021 May;27(5):904-16.

32. Su Y, Chen D, Yuan D, Lausted C, Choi J, Dai CL, et al. Multi-Omics Resolves a Sharp Disease-State Shift between Mild and Moderate COVID-19. Cell. 2020 Dec 10;183(6):147995.e20.

33. Zandi K, Musall K, Oo A, Cao D, Liang B, Hassandarvish P, et al. Baicalein and Baicalin Inhibit SARS-CoV-2 RNA-Dependent-RNA Polymerase. Microorganisms. 2021 Apr 22;9(5).

34. Kärber G. Tabellen zur näherungsweisen Bestimmung von Titern. NaunynSchmiedeberg's Arch exper Path Pharmakol. 1931;162:480.

35. Reed LJ, Muench H. A simple method of estimating fifty per cent endpoints. American journal of epidemiology. 1938;27(3):493-7.

36. Ramakrishnan MA. Determination of $50 \%$ endpoint titer using a simple formula. World J Virol. 2016 May 12;5(2):85-6.

37. Stuyver LJ, Lostia S, Adams M, Mathew JS, Pai BS, Grier J, et al. Antiviral activities and cellular toxicities of modified 2',3'-dideoxy-2',3'-didehydrocytidine analogues. Antimicrob Agents Chemother. 2002 Dec;46(12):3854-60. 
628

38. Chou T-C, Talalay P. Quantitative analysis of dose-effect relationships: the combined effects of multiple drugs or enzyme inhibitors. Advances in enzyme regulation. 1984;22:27-55. 39. Benjamini Y, Krieger AM, Yekutieli D. Adaptive linear step-up procedures that control the false discovery rate. Biometrika. 2006;93(3):491-507.

40. Dobin A, Davis CA, Schlesinger F, Drenkow J, Zaleski C, Jha S, et al. STAR: ultrafast universal RNA-seq aligner. Bioinformatics. 2013 Jan 1;29(1):15-21.

41. Stuart T, Butler A, Hoffman P, Hafemeister C, Papalexi E, Mauck WM, 3rd, et al. Comprehensive Integration of Single-Cell Data. Cell. 2019 Jun 13;177(7):1888-902.e21. 42. Kim D, Lee JY, Yang JS, Kim JW, Kim VN, Chang H. The Architecture of SARS-CoV-2 Transcriptome. Cell. 2020 May 14;181(4):914-21.e10.

43. Lu R, Zhao X, Li J, Niu P, Yang B, Wu H, et al. Genomic characterisation and epidemiology of 2019 novel coronavirus: implications for virus origins and receptor binding. The lancet. 2020;395(10224):565-74.

44. Perkins SM, Webb DL, Torrance SA, El Saleeby C, Harrison LM, Aitken JA, et al. Comparison of a real-time reverse transcriptase PCR assay and a culture technique for quantitative assessment of viral load in children naturally infected with respiratory syncytial virus. J Clin Microbiol. 2005 May;43(5):2356-62.

45. Cook D, Mandell L. Endotracheal aspiration in the diagnosis of ventilator-associated pneumonia. Chest. 2000 Apr;117(4 Suppl 2):195s-7s.

46. Scholte JB, van Dessel HA, Linssen CF, Bergmans DC, Savelkoul PH, Roekaerts PM, et al. Endotracheal aspirate and bronchoalveolar lavage fluid analysis: interchangeable diagnostic modalities in suspected ventilator-associated pneumonia? J Clin Microbiol. 2014 Oct;52(10):3597-604.

47. Kurth J, Waldmann R, Heith J, Mausbach K, Burian R. Efficient inactivation of viruses and mycoplasma in animal sera using UVC irradiation. Dev Biol Stand. 1999;99:111-8. 48. Terpstra FG, van 't Wout AB, Schuitemaker H, van Engelenburg FA, Dekkers DW, Verhaar R, et al. Potential and limitation of UVC irradiation for the inactivation of pathogens in platelet concentrates. Transfusion. 2008 Feb;48(2):304-13.

49. Simón-Manso Y, Lowenthal MS, Kilpatrick LE, Sampson ML, Telu KH, Rudnick PA, et al. Metabolite profiling of a NIST Standard Reference Material for human plasma (SRM 1950): GCMS, LC-MS, NMR, and clinical laboratory analyses, libraries, and web-based resources. Anal Chem. 2013 Dec 17;85(24):11725-31.

50. de Jager TL, Cockrell AE, Du Plessis SS. Ultraviolet Light Induced Generation of Reactive Oxygen Species. Adv Exp Med Biol. 2017;996:15-23.

51. Gruber J, Tang SY, Jenner AM, Mudway I, Blomberg A, Behndig A, et al. Allantoin in human plasma, serum, and nasal-lining fluids as a biomarker of oxidative stress: avoiding artifacts and establishing real in vivo concentrations. Antioxid Redox Signal. 2009 Aug;11(8):1767-76.

52. Lee BC, Gladyshev VN. The biological significance of methionine sulfoxide stereochemistry. Free Radic Biol Med. 2011 Jan 15;50(2):221-7.

53. Maghzal GJ, Leck MC, Collinson E, Li C, Stocker R. Limited role for the bilirubinbiliverdin redox amplification cycle in the cellular antioxidant protection by biliverdin reductase. $J$ Biol Chem. 2009 Oct 23;284(43):29251-9.

54. Chattopadhyay PK, Roederer M. Cytometry: today's technology and tomorrow's horizons. Methods. 2012 Jul;57(3):251-8.

55. Perfetto SP, Chattopadhyay PK, Roederer M. Seventeen-colour flow cytometry: unravelling the immune system. Nat Rev Immunol. 2004 Aug;4(8):648-55.

56. Roederer M, De Rosa S, Gerstein R, Anderson M, Bigos M, Stovel R, et al. 8 color, 10parameter flow cytometry to elucidate complex leukocyte heterogeneity. Cytometry. $1997 \mathrm{Dec}$ 1;29(4):328-39. 
678 57. Woodruff MC, Ramonell RP, Nguyen DC, Cashman KS, Saini AS, Haddad NS, et al. 679 Extrafollicular B cell responses correlate with neutralizing antibodies and morbidity in COVID680 19. Nat Immunol. 2020 Dec;21(12):1506-16.

681 58. Silvin A, Chapuis N, Dunsmore G, Goubet AG, Dubuisson A, Derosa L, et al. Elevated 682 Calprotectin and Abnormal Myeloid Cell Subsets Discriminate Severe from Mild COVID-19. Cell. 6832020 Sep 17;182(6):1401-18.e18.

684 59. Wang J, Mauser A, Chao SF, Remington K, Treckmann R, Kaiser K, et al. Virus 685 inactivation and protein recovery in a novel ultraviolet-C reactor. Vox Sang. 2004

686 May;86(4):230-8.

687 60. Cutts TA, Cook BWM, Poliquin PG, Strong JE, Theriault SS. Correction for Cutts et al., 688 "Inactivating Zaire Ebolavirus in Whole-Blood Thin Smears Used for Malaria Diagnosis". J Clin 689 Microbiol. 2021 May 19;59(6).

690 61. Mok CK, Goh VSL, Ma L, Chu JJH. Establish a standard inactivation protocol for virus 691 research in a high containment laboratory. International Journal of Infectious Diseases.

692 2020;101:254. 
696

A

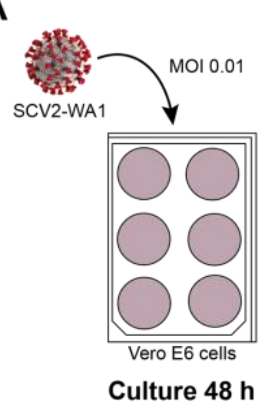

B

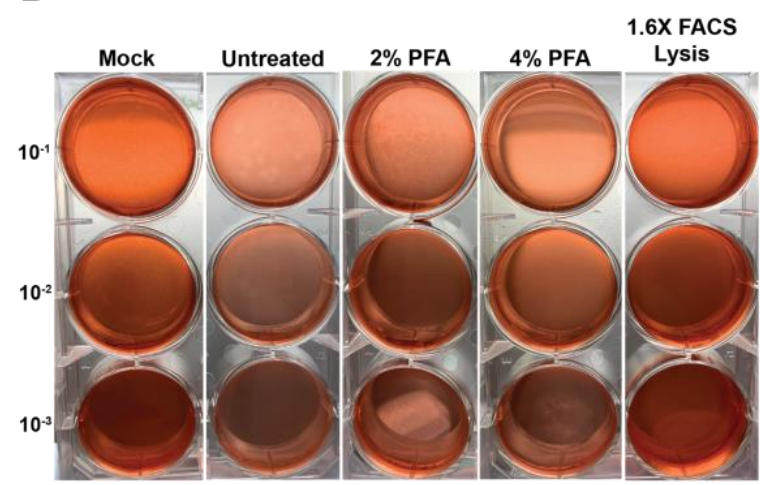

Conditions:

1. Untreated (1X HBSS)

2. $2 \%$ PFA

3. $4 \%$ PFA

4. 1.6X BD ${ }^{\mathrm{TM}}$ FACS Lysis

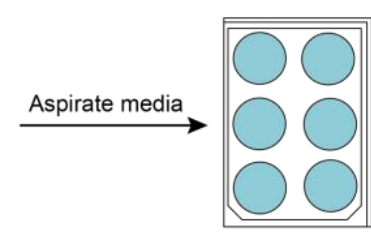

Incubate for: 15-60 mins @ RT

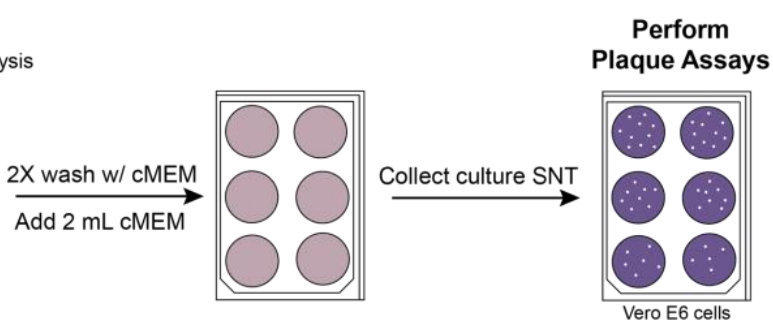

Culture $48 \mathrm{~h}$

C

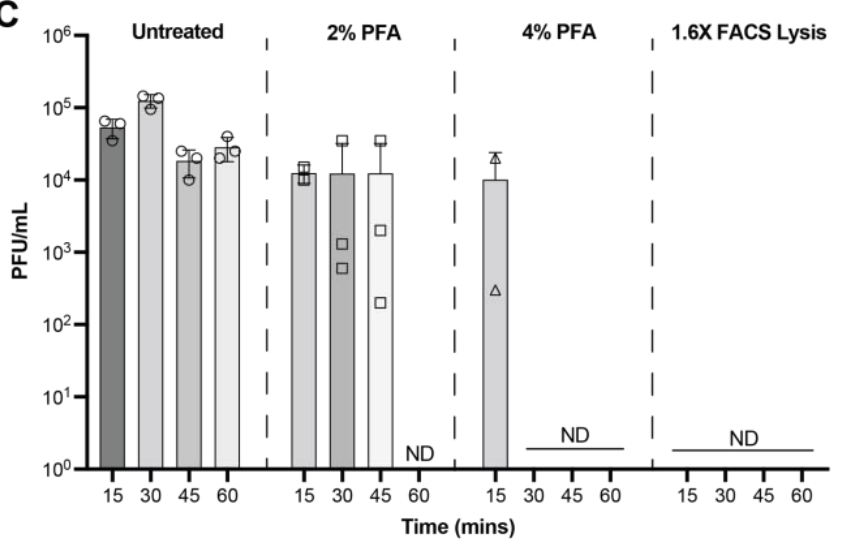

697

698

699

700

701

702

703

704

705

706

707
Figure 1. Fixation with commercially-available fixatives promote complete inactivation of SARS-CoV-2-infected cells amenable to flow cytometric analyses.

A. Schematic of inactivation time course performed to evaluate inactivation efficiency. SNT = supernatant.

B. Representative plaque assays from inactivation time course.

C. Quantification of viral load for the 4 fixatives across the 4 time-points evaluated. ND $=$ Not Detected (by plaque assay). 
bioRxiv preprint doi: https://doi.org/10.1101/2021.10.22.465481; this version posted October 24, 2021. The copyright holder for this preprint (which was not certified by peer review) is the author/funder. All rights reserved. No reuse allowed without permission.

A

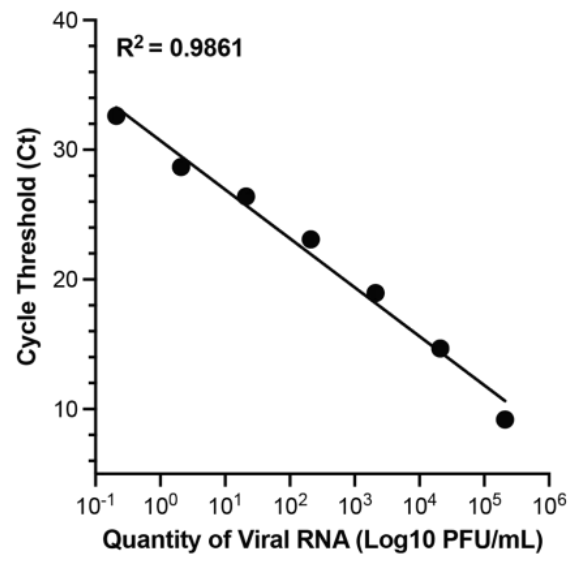

B

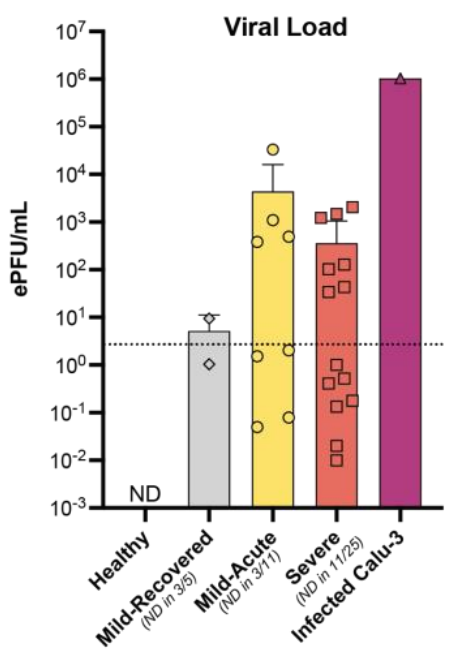

C

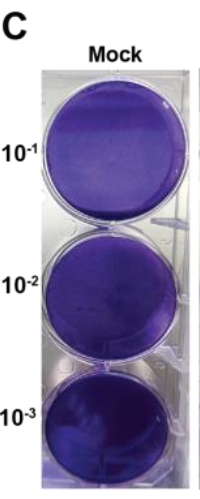

D

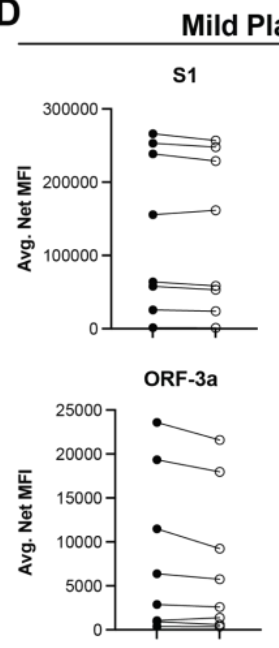

N

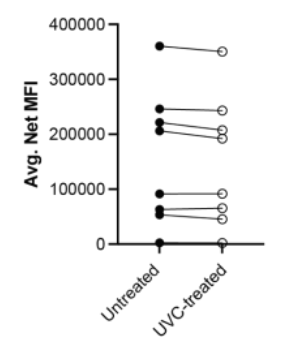

SCV-2-WA1 Viral stock

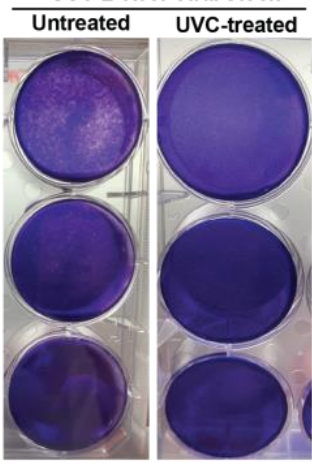

Mild Plasma

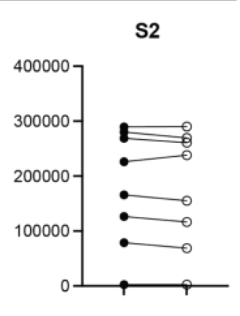

NTD

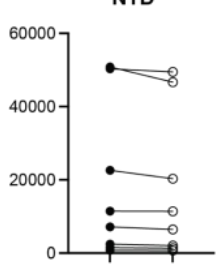

RBD

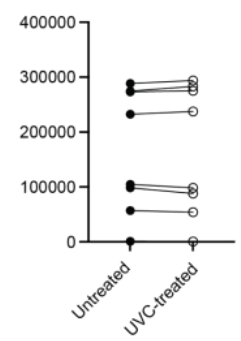

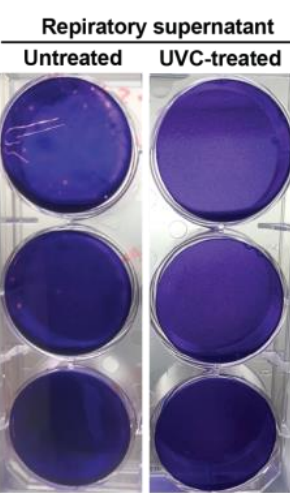

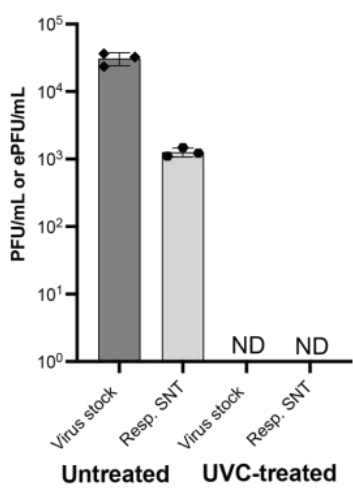

Severe Plasma
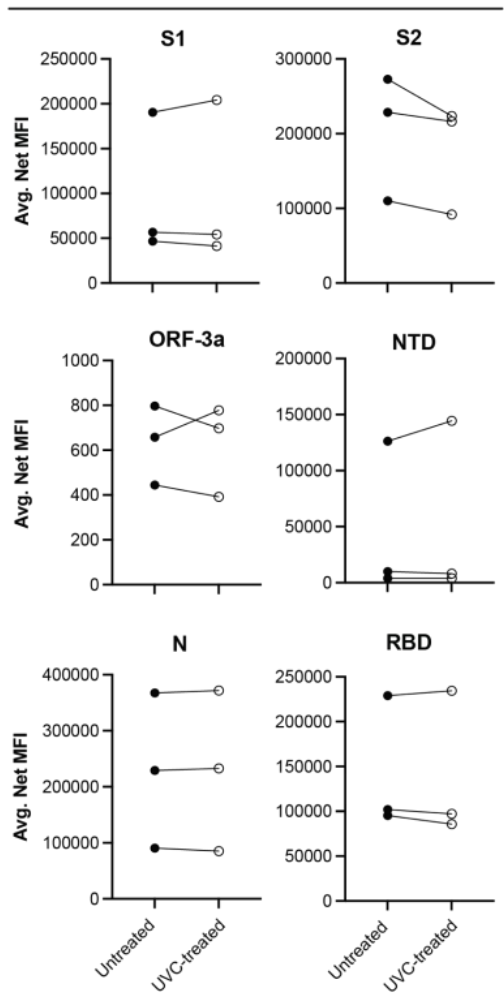
Figure 2. UVC irradiation exposure for 30 minutes inactivates SARS-CoV-2 with minimal effects on antibody/protein detection assays.

A. Viral curve generated from serially-diluted SCV2-WA1 stock of a known titer to extrapolate ePFU/mL from RT-qPCR data.

B. Viral load (ePFU/mL) in respiratory supernatant (Resp. SNT) from non-induced sputum (healthy and mild) and endotracheal aspirates (ETA; severe) samples using the viral curve generated in $A$. Dotted line = lower limit of quantification for the ePFU conversion determined by lowest dilution of stock virus $\left(10^{-6}\right)$ detected by RT-qPCR. ND = Not Detected (by RT-qPCR).

C. Representative plaque assays from stock SCV2-WA1 virus and respiratory supernatant (Resp. SNT) samples and quantification of viral load (PFU/mL for SCV2-WA1 stock and ePFU/mL for Resp. SNT from B) before and after UVC-treatment (30 min at $\sim 4000$ $\mu w a t t / \mathrm{cm}^{2}$ ). ND = Not Detected (by plaque assay).

D. Comparison of SARS-CoV-2 antibody measurements in untreated and UVC-treated plasma samples from mild and severe COVID-19 patients. 
A

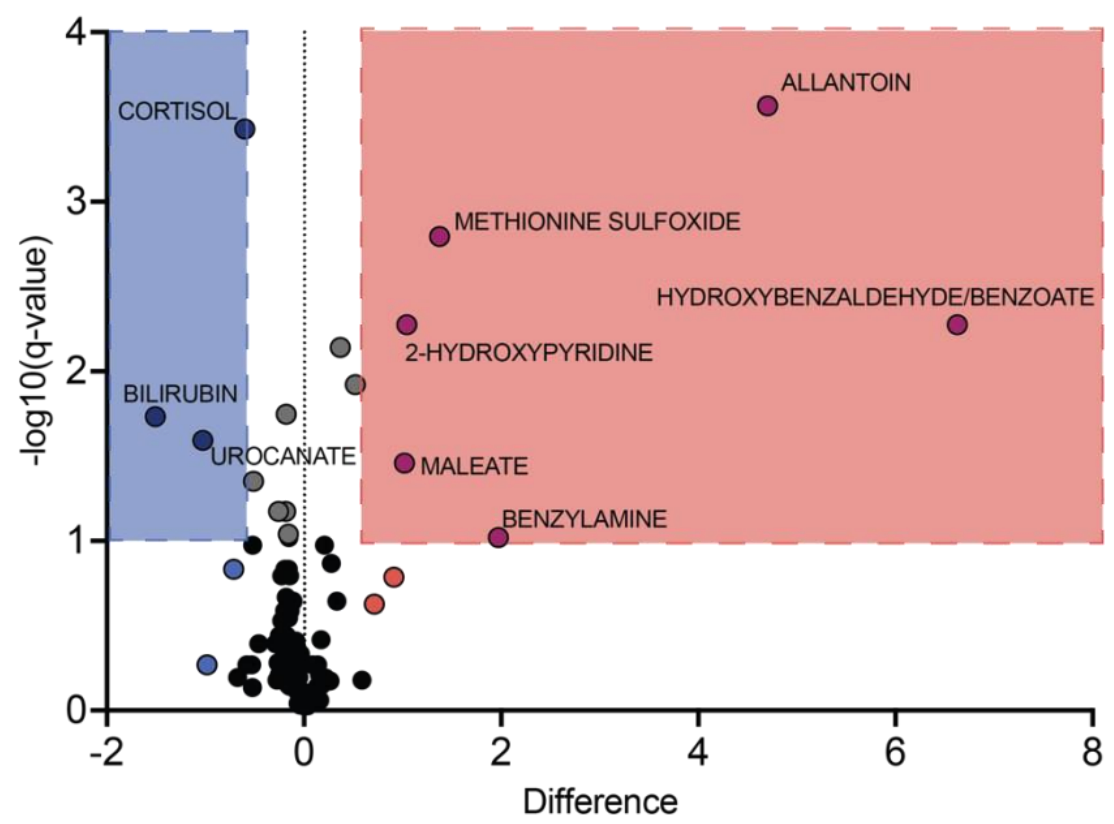

B Allantoin

Methionine Sulfoxide

\section{Bilirubin}
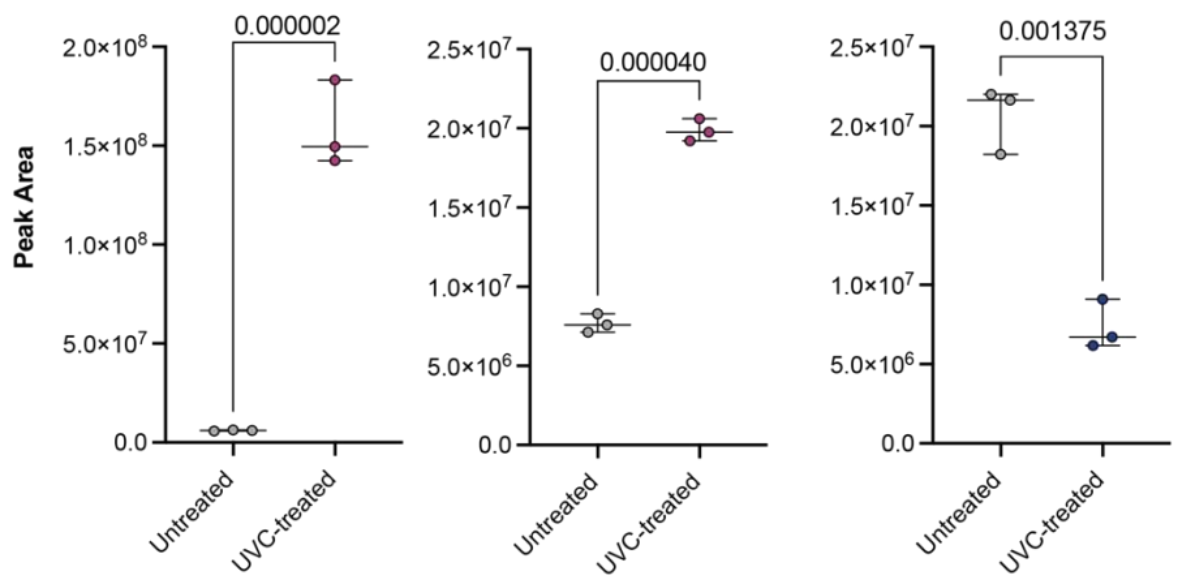

C

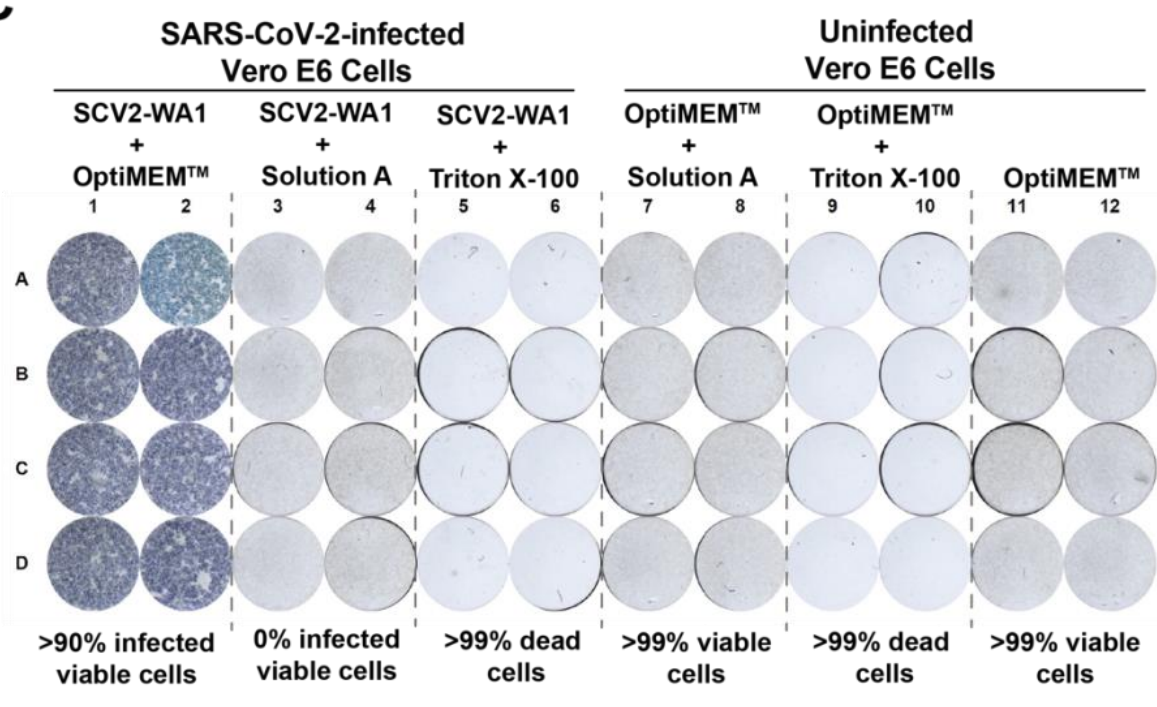


727 Figure 3. Metabolite extraction solvent (Solution A) completely inactivates SARS-CoV-2 728 and maintains sample quality for downstream metabolomics assays.

A. Volcano plot (FDR <10\%) displaying differentially-expressed metabolites in untreated versus UVC-treated NIST (standard) plasma samples.

B. Example plots of 3 representatives differentially-expressed, redox-active metabolites in untreated vs. UVC-treated NIST plasma samples.

C. FRNA results evaluating inactivation of the metabolite extraction solvent (Solution $A$ ) in the standard metabolomic sample processing procedure (see methods) and Triton X-100. 
A $10 x$ reagents
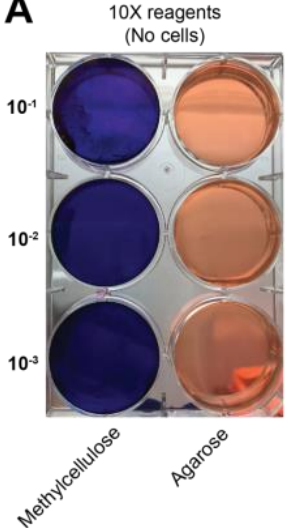

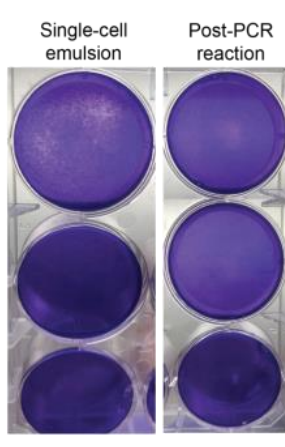

Figure 4. Heat inad
B

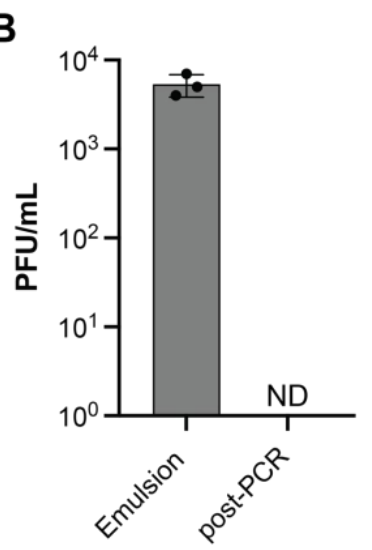

C

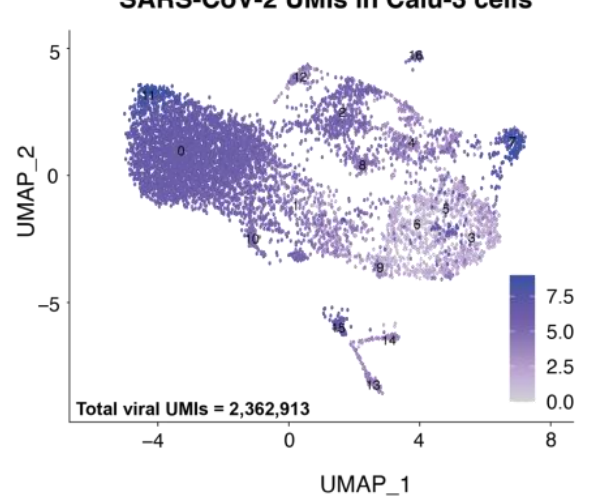

A. Representative plaque assays performed using 10X Genomics' emulsion reagents alone (without cells) to evaluate reagent cytotoxicity on Vero E6 cells and single-cell emulsion with SCV2-WA1-infected Vero E6 cells (MOI 0.04), and the same emulsion after cDNA synthesis PCR reaction ( $45 \mathrm{~min}$ at $53^{\circ} \mathrm{C}$ followed by $5 \mathrm{~min}$ at $85^{\circ} \mathrm{C}$ ).

B. Quantification of viral load in single-cell emulsions of SCV2-WA1-infected Calu-3 cells ( $\mathrm{MOI}$ 0.04) immediately after encapsulation and following PCR reaction for cDNA synthesis. $\mathrm{N}=3$ independent samples. $\mathrm{ND}=$ Not Detected (by plaque assay).

C. UMAP visualization of scRNA-seq data from SCV2-WA1-infected Calu-3 cells (MOI 0.04; $\mathrm{N}=8061$ cells) showing expression of the 12 SARS-CoV-2 genes and total viral UMls (inset). 
Untreated

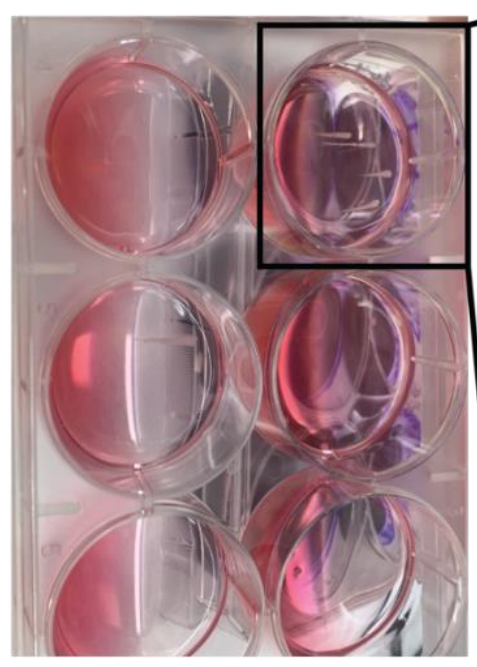

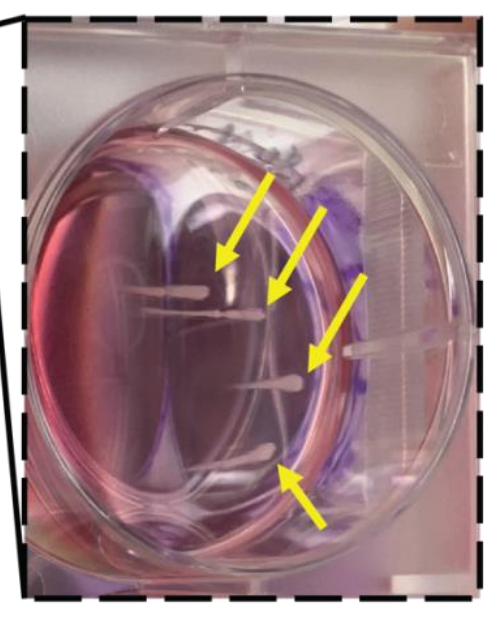

UVC-treated

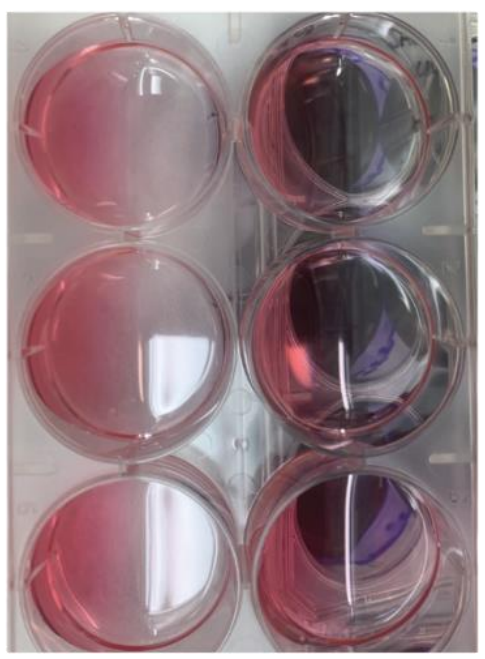

Figure S1. UVC inactivation abolishes microbial growth in plaque assay cultures.

Representative image of plaque assay cultures for respiratory supernatant samples (severe COVID-19 patients) before and after UVC-treatment (30 min at $\sim 4000 \mu \mathrm{watt} / \mathrm{cm}^{2}$ ). Yellow arrows 755 indicate microbial growth. 